\title{
Nonlinear Global Stabilization Control for the Underactuated WAcrobot System
}

\author{
Shuli Gong $\mathbb{D}^{1},{ }^{1}$ Ancai Zhang $\mathbb{D}^{2,3}$ Zhi Liu, ${ }^{2,3}$ Zhenxing Li $\mathbb{D}^{2,3}$ Chengdong Yang, \\ and Xinghui Zhang ${ }^{2,3}$ \\ ${ }^{1}$ School of Mathematics and Statistics, Linyi University, Linyi, Shandong 276000, China \\ ${ }^{2}$ School of Automation and Electrical Engineering, Linyi University, Linyi, Shandong 276000, China \\ ${ }^{3}$ Key Laboratory of Complex Systems and Intelligent Computing in Universities of Shandong, Linyi, Shandong 276000, China \\ ${ }^{4}$ School of Information Science and Engineering, Linyi University, Linyi, Shandong 276000, China
}

Correspondence should be addressed to Ancai Zhang; zhangancai123@hotmail.com

Received 20 April 2020; Accepted 9 June 2020; Published 3 July 2020

Guest Editor: Ping Zhao

Copyright (c) 2020 Shuli Gong et al. This is an open access article distributed under the Creative Commons Attribution License, which permits unrestricted use, distribution, and reproduction in any medium, provided the original work is properly cited.

A WAcrobot is an underactuated nonlinear system that has three degrees of freedom (DOF) and two inputs. This paper discusses the global stabilization control problem for this 3-DOF underactuated system. A new control strategy is developed to solve this problem. The strategy first changes the 3-DOF WAcrobot system to be a 2-DOF reduced-order model in finite time. This transforms the stabilizing control of the WAcrobot system into that of the reduced-order model. After that, nonsingular control laws that globally stabilize the reduced-order model at the origin are designed. It guarantees the stabilizing control objective of the WAcrobot to be achieved. Finally, a simulation experimental example demonstrates the validity of the presented theoretical results. Simulation results show the advantage of our strategy over others.

\section{Introduction}

In our daily life, many natural systems essentially have nonlinear characteristics. It is meaningful to discuss the control problem for the nonlinear systems [1-5]. An underactuated mechanical system is a typical example of nonlinear systems, which has fewer control inputs than the number of systems' degrees of freedom (DOF). Compared with the fully actuated system, the underactuated system has the characteristics of light weight, low energy consumption, and flexible movement because of the reduction of actuators. Such systems can be widely used in health care, space exploration, transportation, military, and other fields [6-9]. However, the reduction of inputs makes the system possess nonlinear constraints. And the constraints are usually second-order nonholonomic [10]. This means that the states of the system are in uncontrollable manifolds of the configuration space. The control design of an underactuated mechanical system is challenging in the nonlinear control area. Many researchers have devoted their efforts to the study on underactuated systems in the past few years [11-15].

Since the 1990s, the control problems for the simplest underactuated system (that is, the 2-DOF underactuated system) have been attracting much attention. In order to explore the nonlinear control strategy, many experimental models of 2-DOF underactuated systems have been presented in the laboratory. The models include Acrobot [16], Pendubot [17], TORA [18], and Pendulum-cart [19]. The stabilization control issue is a commonly addressed problem for these 2-DOF underactuated mechanical systems. And many stabilizing control methods have been developed, e.g., a partial feedback linearization method [20], an energybased method [21], a nonsmooth Lyapunov function method [22], an equivalent-input disturbance method [23], and a trajectory tracking strategy [24].

With the deepening of the research work, the control problems presented by an $n$-DOF $(n \geq 3)$ underactuated system need to be further explored since multi-DOF systems are more consistent with the reality of natural systems. 
However, this problem is not easy to solve because the nonlinear dynamics and constraint equations of underactuated systems become more complicated with the increase of the DOF. To solve the control problems for multiDOF underactuated systems, it is necessary to study the simplest case, i.e., $n=3$. Recently, some 3-DOF underactuated system models have been presented. Their dynamic analysis and control design have been intensively discussed [25-28]. Among them, a WAcrobot model (see Figure 1) is a typical example. This mechanical system has a wheel and a double inverted pendulum. The wheel rolls in a horizontal plane driven by an actuator. And the double pendulum freely rotates in a vertical plane driven by an actuator at the second joint. The first joint of the pendulum is passive. Note that the WAcrobot is a 3-DOF underactuated system, and it is the combination of a wheel and an Acrobot.

The WAcrobot has a good application prospect in the mobile robotic area. It is a perfect test bed for illustrating a nonlinear control algorithm for multi-DOF underactuated systems. So, it is meaningful to study the problems presented by this mechanical system. In [29], the swing-up and stabilization control of the WAcrobot were discussed. The authors used noncollocated feedback linearization and linear quadratic regulator technique to achieve the system's control objective. In addition to this paper, there are no related research results about this system. Since the WAcrobot possesses common inherent nonlinear features of multiDOF underactuated systems, it is necessary to explore more control methods for it. This inspires our study in this paper.

The global stabilization control problem for the WAcrobot is addressed in this paper. By using the inherent dynamic coupling relationship, we first change the WAcrobot into a 2-DOF reduced-order system in finite time. And then, the global stabilization of the reduced-order model is discussed. Stabilizing control laws are developed, and the condition that avoids the singularity in the control law is also presented. Finally, numerical experiments demonstrate the validity of the theoretical analysis results. Simulation results show the advantage of our strategy over the results in [29]. Moreover, the presented strategy gives a good guidance for the solution of motion control of other multi-DOF underactuated mechanical systems.

\section{Dynamic Motion Equations of the WAcrobot System}

For the physical model of a WAcrobot system shown in Figure $1, \theta_{1}(t), m_{1}, J_{1}$, and $L_{1}$ are the rotational angle, the mass, the moment of inertia, and the radius of the wheel, respectively. For $i=2,3, \theta_{1}(t), m_{i}, J_{i}$, and $L_{i}$ are the rotational angle, the mass, the moment of inertia, and the length of the $i$ th pendulum, respectively, and $L_{c i}$ is the distance from the $i$ th joint to the center of mass (COM) of the $i$ th pendulum. Moreover, $\tau_{1}(t)$ and $\tau_{2}(t)$ are the input torques applied on the wheel and the 2nd pendulum, respectively, and $g$ is the gravity acceleration constant $\left(\approx 9.81 \mathrm{~m} / \mathrm{s}^{2}\right)$.

We take the plane in which the center of the circle is located on as the zero potential energy reference plane. A

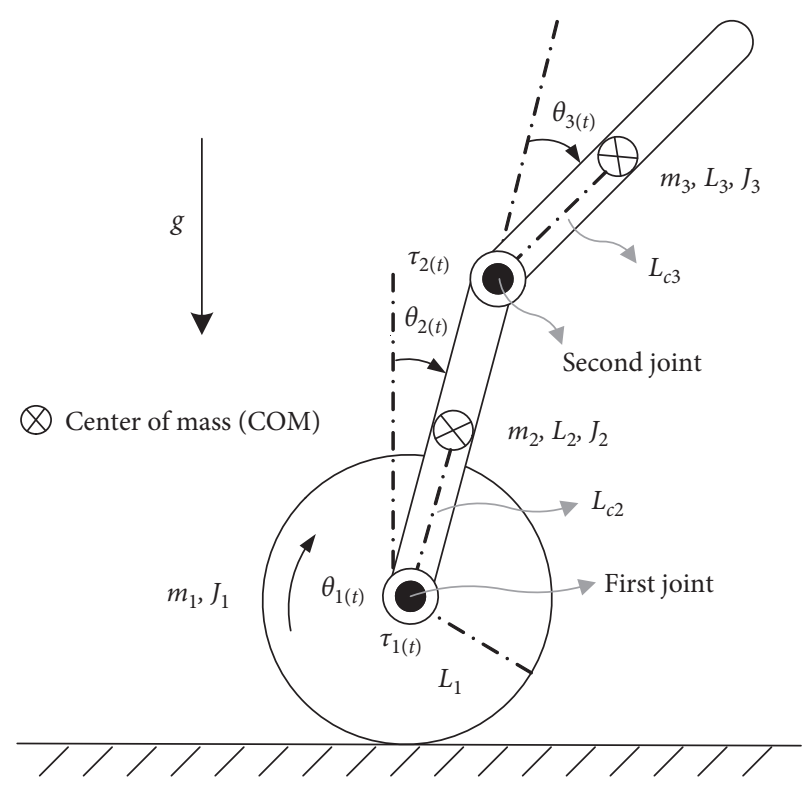

Figure 1: Physical model of the WAcrobot system.

simple calculation gives the potential energy of the WAcrobot as

$$
\left\{\begin{array}{l}
P(\boldsymbol{\theta})=\beta_{1} \cos \theta_{2}+\beta_{2} \cos \left(\theta_{2}+\theta_{3}\right), \\
\beta_{1}=\left(m_{2} L_{c 2}+m_{3} L_{2}\right) g, \\
\beta_{2}=m_{3} L_{3} g,
\end{array}\right.
$$

where $\theta=\left[\theta_{1}, \theta_{2}, \theta_{3}\right]^{\top}$. In addition, the kinetic energy of the system is

$$
K(\boldsymbol{\theta}, \dot{\theta})=\frac{1}{2} \dot{\theta}^{\top} M(\boldsymbol{\theta}) \dot{\theta}
$$

where

$$
\begin{aligned}
& M(\boldsymbol{\theta})=\left[\begin{array}{ccc}
M_{11} & M_{12}(\boldsymbol{\theta}) & M_{13}(\boldsymbol{\theta}) \\
M_{21}(\boldsymbol{\theta}) & M_{22}(\boldsymbol{\theta}) & M_{23}(\boldsymbol{\theta}) \\
M_{31}(\boldsymbol{\theta}) & M_{32}(\boldsymbol{\theta}) & M_{33}
\end{array}\right], \\
& \left\{\begin{array}{l}
M_{11}=\alpha_{1}, M_{22}(\boldsymbol{\theta})=\alpha_{2}+\alpha_{4}+2 \alpha_{6} \cos \theta_{3}, \\
M_{33}=\alpha_{4}, \\
M_{12}(\boldsymbol{\theta})=M_{21}(\boldsymbol{\theta})=\alpha_{3} \cos \theta_{2}+\alpha_{5} \cos \left(\theta_{2}+\theta_{3}\right),
\end{array}\right. \\
& M_{13}(\boldsymbol{\theta})=M_{31}(\boldsymbol{\theta})=\alpha_{5} \cos \left(\theta_{2}+\theta_{3}\right) \text {, } \\
& M_{23}(\boldsymbol{\theta})=M_{32}(\boldsymbol{\theta})=\alpha_{4}+\alpha_{6} \cos \theta_{2}, \\
& \left\{\alpha_{1}=\left(m_{1}+m_{2}+m_{3}\right) L_{1}^{2}+J_{1},\right. \\
& \alpha_{2}=m_{2} L_{c 2}^{2}+J_{2}+m_{3} L_{2}^{2} \text {, } \\
& \alpha_{3}=\left(m_{2} L_{c 2}+m_{3} L_{2}\right) L_{1} \text {, } \\
& \alpha_{4}=m_{3} L_{c 3}^{2}+J_{3} \text {, } \\
& \alpha_{5}=m_{3} L_{1} L_{c 3} \text {, } \\
& \alpha_{6}=m_{3} L_{2} L_{c 3} \text {. }
\end{aligned}
$$

The Lagrangian function of the WAcrobot system is taken to be $L(\theta, \dot{\theta})=K(\theta, \dot{\theta})-P(\theta)$. We get the Euler-Lagrange motion equations of the system as 


$$
\left\{\begin{array}{l}
\frac{\mathrm{d}}{\mathrm{d} t}\left[\frac{\partial L(\boldsymbol{\theta}, \dot{\theta})}{\partial \dot{\theta}_{1}}\right]-\frac{\partial L(\boldsymbol{\theta}, \dot{\theta})}{\partial \theta_{1}}=\tau_{1}, \\
\frac{\mathrm{d}}{\mathrm{d} t}\left[\frac{\partial L(\boldsymbol{\theta}, \dot{\theta})}{\partial \dot{\theta}_{2}}\right]-\frac{\partial L(\boldsymbol{\theta}, \dot{\theta})}{\partial \theta_{2}}=0, \\
\frac{\mathrm{d}}{\mathrm{d} t}\left[\frac{\partial L(\boldsymbol{\theta}, \dot{\theta})}{\partial \dot{\theta}_{3}}\right]-\frac{\partial L(\boldsymbol{\theta}, \dot{\theta})}{\partial \theta_{3}}=\tau_{2} .
\end{array}\right.
$$

These dynamic equations can be rewritten in the following form:

$$
M(\boldsymbol{\theta}) \ddot{\theta}+\mathbf{H}(\boldsymbol{\theta}, \dot{\theta})+\mathbf{G}(\boldsymbol{\theta})=\boldsymbol{\tau},
$$

where

$$
\begin{aligned}
\mathbf{H}(\boldsymbol{\theta}, \dot{\theta}) & =\left[\begin{array}{l}
H_{1}(\boldsymbol{\theta}, \dot{\theta}) \\
H_{2}(\boldsymbol{\theta}, \dot{\theta}) \\
H_{3}(\boldsymbol{\theta}, \dot{\theta})
\end{array}\right], \\
\mathbf{G}(\boldsymbol{\theta}) & =\left[\begin{array}{c}
0 \\
G_{2}(\boldsymbol{\theta}) \\
G_{3}(\boldsymbol{\theta})
\end{array}\right], \\
\boldsymbol{\tau} & =\left[\begin{array}{c}
\tau_{1} \\
0 \\
\tau_{2}
\end{array}\right], \\
& \left\{\begin{array}{l}
H_{1}(\boldsymbol{\theta}, \dot{\theta})=-\alpha_{3} \dot{\theta}_{2}^{2} \sin \theta_{2}-\alpha_{5}\left(\dot{\theta}_{2}+\dot{\theta}_{3}\right)^{2} \sin \left(\theta_{2}+\theta_{3}\right), \\
H_{2}(\boldsymbol{\theta}, \dot{\theta})=-\alpha_{6}\left(2 \dot{\theta}_{2}+\dot{\theta}_{3}\right) \dot{\theta}_{3} \sin \theta_{3}, \\
H_{3}(\boldsymbol{\theta}, \dot{\theta})=\alpha_{6} \dot{\theta}_{2}^{2} \sin \theta_{3}, \\
G_{2}(\boldsymbol{\theta})=-\beta_{1} \sin \theta_{2}-\beta_{2} \sin \left(\theta_{2}+\theta_{3}\right), \\
G_{3}(\boldsymbol{\theta})=-\beta_{2} \sin \left(\theta_{2}+\theta_{3}\right) .
\end{array}\right.
\end{aligned}
$$

Denote $\Xi(\theta, \dot{\theta})=K(\theta, \dot{\theta})+P(\theta)$ to be the total energy of the WAcrobot. From (5), we easily obtain

$$
\dot{\Xi}(\boldsymbol{\theta}, \dot{\theta})=\dot{\theta}^{\top} \tau=\dot{\theta}_{1} \tau_{1}+\dot{\theta}_{3} \tau_{2} .
$$

Let $x_{1}=\theta_{1}, x_{2}=\theta_{1}, x_{3}=\theta_{2}, x_{4}=\theta_{2}, z_{1}=\theta_{3}, z_{2}=\dot{\theta}_{3}$, $\mathbf{x}=\left[x_{1}, x_{2}, x_{3}, x_{4}\right]^{\top}$, and $\mathbf{z}=\left[z_{1}, z_{2}\right]^{\top}$. Then, it is not difficult to obtain the state space form of (5) as

$$
\left\{\begin{array}{l}
\dot{x}_{1}=x_{2} \\
\dot{x}_{2}=\Upsilon_{1}(\mathbf{x}, \mathbf{z})+\Phi_{1}(\mathbf{x}, \mathbf{z}) \tau_{1}+\Psi_{1}(\mathbf{x}, \mathbf{z}) \tau_{2}, \\
\dot{x}_{3}=x_{4} \\
\dot{x}_{4}=\Upsilon_{2}(\mathbf{x}, \mathbf{z})+\Phi_{2}(\mathbf{x}, \mathbf{z}) \tau_{1}+\Psi_{2}(\mathbf{x}, \mathbf{z}) \tau_{2}, \\
\dot{z}_{1}=z_{2} \\
\dot{z}_{2}=\Upsilon_{3}(\mathbf{x}, \mathbf{z})+\Phi_{3}(\mathbf{x}, \mathbf{z}) \tau_{1}+\Psi_{3}(\mathbf{x}, \mathbf{z}) \tau_{2}
\end{array}\right.
$$

where

$$
\begin{aligned}
& {\left[\begin{array}{l}
\Upsilon_{1}(\mathbf{x}, \mathbf{z}) \\
\Upsilon_{2}(\mathbf{x}, \mathbf{z}) \\
\Upsilon_{3}(\mathbf{x}, \mathbf{z})
\end{array}\right]=M^{-1}(\boldsymbol{\theta})\left[\begin{array}{c}
-H_{1}(\boldsymbol{\theta}, \dot{\theta}) \\
-H_{2}(\boldsymbol{\theta}, \dot{\theta})-G_{2}(\boldsymbol{\theta}) \\
-H_{3}(\boldsymbol{\theta}, \dot{\theta})-G_{3}(\boldsymbol{\theta})
\end{array}\right],} \\
& {\left[\begin{array}{ll}
\Phi_{1}(\mathbf{x}, \mathbf{z}) & \Psi_{1}(\mathbf{x}, \mathbf{z}) \\
\Phi_{2}(\mathbf{x}, \mathbf{z}) & \Psi_{2}(\mathbf{x}, \mathbf{z}) \\
\Phi_{3}(\mathbf{x}, \mathbf{z}) & \Psi_{3}(\mathbf{x}, \mathbf{z})
\end{array}\right]=M^{-1}(\boldsymbol{\theta})\left[\begin{array}{ll}
1 & 0 \\
0 & 0 \\
0 & 1
\end{array}\right] .}
\end{aligned}
$$

It follows from (9) that both $\Psi_{3}(\mathbf{x}, \mathbf{z})$ and $\Phi_{1}(\mathbf{x}, \mathbf{z}) \Psi_{3}(\mathbf{x}, \mathbf{z})-\Phi_{3}(\mathbf{x}, \mathbf{z}) \Psi_{1}(\mathbf{x}, \mathbf{z})$ are order principal determinants of $M^{-1}(\theta)$. Since $M^{-1}(\theta)$ is a positive definite matrix, we get

$$
\left\{\begin{array}{l}
\Psi_{3}(\mathbf{x}, \mathbf{z})>0, \\
\Phi_{1}(\mathbf{x}, \mathbf{z}) \Psi_{3}(\mathbf{x}, \mathbf{z})-\Phi_{3}(\mathbf{x}, \mathbf{z}) \Psi_{1}(\mathbf{x}, \mathbf{z})>0 .
\end{array}\right.
$$

\section{Reduced-Order Model of the WAcrobot System}

The control objective discussed in this paper is to design controllers $\tau_{1}$ and $\tau_{2}$ to globally stabilize WAcrobot system (8) at $[\mathbf{x}, \mathbf{z}]^{\top}=\mathbf{0}$. Note that the nonlinear dynamics of (8) is very complicated. In order to simplify the structure of system (8), we design

$$
\tau_{2}=\frac{W\left(z_{1}, z_{2}\right)-\Upsilon_{3}(\mathbf{x}, \mathbf{z})-\Phi_{3}(\mathbf{x}, \mathbf{z}) \tau_{1}}{\Psi_{3}(\mathbf{x}, \mathbf{z})},
$$

where

$$
W\left(z_{1}, z_{2}\right)=-r_{2}\left(z_{2}^{5 / 3}+r_{1}^{5 / 3} z_{1}\right)^{1 / 5}
$$

and $r_{1}$ and $r_{2}$ are positive constants. This is a relationship between control torques $\tau_{1}$ and $\tau_{2}$. Substituting (11) into (8) yields

$$
\left\{\begin{array}{l}
\dot{x}_{1}=x_{2}, \\
\dot{x}_{2}=\Upsilon_{1}(\mathbf{x}, \mathbf{z})+\frac{\Psi_{1}(\mathbf{x}, \mathbf{z})\left[W\left(z_{1}, z_{2}\right)-\Upsilon_{3}(\mathbf{x}, \mathbf{z})\right]}{\Psi_{3}(\mathbf{x}, \mathbf{z})} \\
+\left[\Phi_{1}(\mathbf{x}, \mathbf{z})-\frac{\Psi_{1}(\mathbf{x}, \mathbf{z}) \Phi_{3}(\mathbf{x}, \mathbf{z})}{\Psi_{3}(\mathbf{x}, \mathbf{z})}\right] \tau_{1}, \\
\dot{x}_{3}=x_{4}, \\
\dot{x}_{4}=\Upsilon_{2}(\mathbf{x}, \mathbf{z})+\frac{\Psi_{2}(\mathbf{x}, \mathbf{z})\left[W\left(z_{1}, z_{2}\right)-\Upsilon_{3}(\mathbf{x}, \mathbf{z})\right]}{\Psi_{3}(\mathbf{x}, \mathbf{z})} \\
+\left[\Phi_{2}(\mathbf{x}, \mathbf{z})-\frac{\Psi_{2}(\mathbf{x}, \mathbf{z}) \Phi_{3}(\mathbf{x}, \mathbf{z})}{\Psi_{3}(\mathbf{x}, \mathbf{z})}\right] \tau_{1}, \\
\left\{\begin{array}{l}
\dot{z}_{1}=z_{2}, \\
\dot{z}_{2}=W\left(z_{1}, z_{2}\right)=-r_{2}\left(z_{2}^{5 / 3}+r_{1}^{5 / 3} z_{1}\right)^{1 / 5} .
\end{array}\right.
\end{array}\right.
$$


It is clear that (13a) and (13b) are a nonlinear cascade system, and the state variable $\mathbf{z}$ is separated from others. According to the results in [30], the finite-time stabilization of subsystem (13b) at $\mathbf{z}=\mathbf{0}$ is achieved. As a result, system (13a) and (13b) becomes the following form in finite time

$$
\left\{\begin{array}{l}
\dot{x}_{1}=x_{2}, \\
\dot{x}_{2}=f_{1}(\mathbf{x})+g_{1}(\mathbf{x}) \tau_{1}, \\
\dot{x}_{3}=x_{4}, \\
\dot{x}_{4}=f_{2}(\mathbf{x})+g_{2}(\mathbf{x}) \tau_{1},
\end{array}\right.
$$

where

$$
\begin{aligned}
& f_{1}(\mathbf{x})=\frac{\Upsilon_{1}(\mathbf{x}, \mathbf{0}) \Psi_{3}(\mathbf{x}, \mathbf{0})-\Upsilon_{3}(\mathbf{x}, \mathbf{0}) \Psi_{1}(\mathbf{x}, \mathbf{0})}{\Psi_{3}(\mathbf{x}, \mathbf{0})}, \\
& f_{2}(\mathbf{x})=\frac{\Upsilon_{2}(\mathbf{x}, \mathbf{0}) \Psi_{3}(\mathbf{x}, \mathbf{0})-\Upsilon_{3}(\mathbf{x}, \mathbf{0}) \Psi_{2}(\mathbf{x}, \mathbf{0})}{\Psi_{3}(\mathbf{x}, \mathbf{0})}, \\
& g_{1}(\mathbf{x})=\frac{\Phi_{1}(\mathbf{x}, \mathbf{0}) \Psi_{3}(\mathbf{x}, \mathbf{0})-\Phi_{3}(\mathbf{x}, \mathbf{0}) \Psi_{1}(\mathbf{x}, \mathbf{0})}{\Psi_{3}(\mathbf{x}, \mathbf{0})} \\
& g_{2}(\mathbf{x})=\frac{\Phi_{2}(\mathbf{x}, \mathbf{0}) \Psi_{3}(\mathbf{x}, \mathbf{0})-\Phi_{3}(\mathbf{x}, \mathbf{0}) \Psi_{2}(\mathbf{x}, \mathbf{0})}{\Psi_{3}(\mathbf{x}, \mathbf{0})}
\end{aligned}
$$

From (10), it is easy to obtain $g_{1}(\mathbf{x})>0$ for $\mathbf{x} \in \mathbb{R}^{4}$.

In fact, nonlinear system (14) is the zero dynamics of cascade system (13a) and (13b). In other words, (14) is a reduced-order system of the WAcrobot. The physical model of (12) is shown in Figure 2. In order to achieve the control objective of the WAcrobot, it is necessary to design a controller $\tau_{1}$ such that system (14) is stabilized at $\mathbf{x}=\mathbf{0}$.

\section{Design of the Stabilizing Controller for the Reduced-Order Model}

This section discusses the design of a stabilizing controller $\tau_{1}$ for reduced-order system (14) under the condition $\mathbf{z}=\mathbf{0}$.

Let $E(x)=\left.\Xi(\theta, \theta)\right|_{\mathbf{z}=0}$. From (1), (2), and (7), it is easy to get $E(0)=\beta_{1}+\beta_{2}$ and $\dot{E}(x)=x_{2} \tau_{1}$. A Lyapunov function for system (14) is constructed to be

$$
V(\mathbf{x})=\frac{\mu_{1}}{2} \widehat{E}^{2}(\mathbf{x})+\frac{\mu_{2}}{2} x_{1}^{2}+\frac{\mu_{3}}{2} x_{2}^{2},
$$

where $\widehat{E}(\mathbf{x})=E(x)-E(0)$ and $\mu_{i}>0(i=1,2,3)$ are constants. The derivative of $V(\mathbf{x})$ is

$$
\begin{aligned}
\frac{\mathrm{d} V(\mathbf{x})}{\mathrm{d} t} & =\mu_{1} \widehat{E}(\mathbf{x}) \dot{E}(x)+\mu_{2} x_{1} \dot{x}_{1}+\mu_{3} x_{2} \dot{x}_{2} \\
& =\mu_{1} \widehat{E}(\mathbf{x}) x_{2} \tau_{1}+\mu_{2} x_{1} x_{2}+\mu_{3} x_{2}\left[f_{1}(\mathbf{x})+g_{1}(\mathbf{x}) \tau_{1}\right] \\
& =\left\{\left[\mu_{1} \widehat{E}(\mathbf{x})+\mu_{3} g_{1}(\mathbf{x})\right] \tau_{1}+\mu_{2} x_{1}+\mu_{3} f_{1}(\mathbf{x})\right\} x_{2} .
\end{aligned}
$$

We design the control law $\tau_{1}$ to be

$$
\tau_{1}=\frac{-\gamma z_{2}-\mu_{2} x_{1}-\mu_{3} f_{1}(\mathbf{x})}{\mu_{1} \widehat{E}(\mathbf{x})+\mu_{3} g_{1}(\mathbf{x})},
$$

where $\gamma>0$ is a constant. Combining (17) and (18) gives

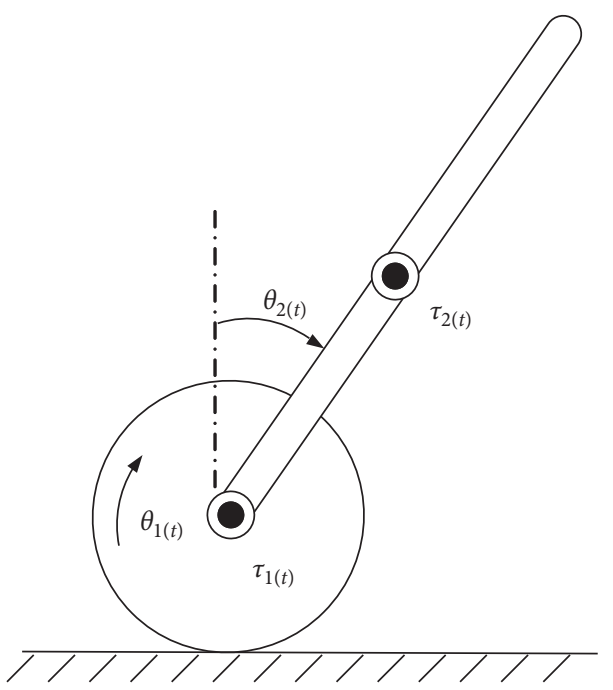

Figure 2: Physical model of (14).

$$
\frac{\mathrm{d} V(\mathbf{x})}{\mathrm{d} t}=-\gamma x_{2}^{2} \leq 0
$$

From Figure 2, it is easy to deduce that system (14) has the same dynamic properties as the Pendubot. As a result, following the same analytical procedure given in Section 4 of [22] yields that $E(x) \longrightarrow E(0), x_{1} \longrightarrow 0$, and $x_{2} \longrightarrow 0$ based on (16) and (19). This means that the control law (18) drives system (14) to converge the set

$$
\begin{aligned}
\Omega & =\left\{\mathbf{x} \mid x_{1}=0, x_{2}=0, E(x)=E(0)\right\} \\
& =\left\{\mathbf{x} \mid x_{1}=0, x_{2}=0, \omega\left(x_{3}, x_{4}\right)=0\right\},
\end{aligned}
$$

where

$$
\omega\left(x_{3}, x_{4}\right)=M_{22}(0) x_{4}^{2}-2\left(\beta_{1}+\beta_{2}\right)\left[1-\cos x_{3}\right] .
$$

Since $x_{4}=\dot{x}_{3}$, we have that $\omega\left(x_{3}, x_{4}\right)=0$ which describes a homoclinic orbit around $x_{3}=x_{4}=0$. Thus, the state variable $\mathbf{x}$ in $\Omega$ can enter any arbitrarily small neighbourhood area of $\mathbf{x}=0$. In this area, system (14) approximates the following linear system:

$$
\dot{x}=A \mathbf{x}+B \tau_{1} \text {, }
$$

where

$$
\begin{aligned}
& A=\left[\begin{array}{llll}
0 & 1 & 0 & 0 \\
0 & 0 & A_{1} & 0 \\
0 & 0 & 0 & 1 \\
0 & 0 & A_{2} & 0
\end{array}\right], \\
& B=\left[\begin{array}{l}
0 \\
B_{1} \\
0 \\
B_{2}
\end{array}\right],
\end{aligned}
$$

where 


$$
\begin{aligned}
A_{1} & =\left.\frac{\partial f_{1}(\mathbf{x})}{\partial x_{3}}\right|_{\mathbf{x}=\mathbf{0}}=\frac{a_{1} c_{3}-a_{3} c_{1}}{c_{3}}, \\
A_{2} & =\left.\frac{\partial f_{2}(\mathbf{x})}{\partial x_{3}}\right|_{\mathbf{x}=\mathbf{0}}=\frac{a_{2} c_{3}-a_{3} c_{2}}{c_{3}}, \\
B_{1} & =\left.g_{1}(\mathbf{x})\right|_{\mathbf{x}=\mathbf{0}}=\frac{b_{1} c_{3}-b_{3} c_{1}}{c_{3}}, \\
B_{2} & =\left.g_{2}(\mathbf{x})\right|_{\mathbf{x}=\mathbf{0}}=\frac{b_{2} c_{3}-b_{3} c_{2}}{c_{3}}, \\
U & =\left[\begin{array}{lll}
a_{1} & b_{1} & c_{1} \\
a_{2} & b_{2} & c_{2} \\
a_{3} & b_{3} & c_{3}
\end{array}\right]=M^{-1}(\mathbf{0})\left[\begin{array}{ccc}
0 & 1 & 0 \\
\beta_{1}+\beta_{2} & 0 & 0 \\
\beta_{2} & 0 & 1
\end{array}\right] .
\end{aligned}
$$

Let $\Lambda=\left[B, A B, A^{2} B, A^{3} B\right]$. It is easy to obtain

$$
\operatorname{det}[\Lambda]=B_{2}^{2}\left[A_{2} B_{1}-A_{1} B_{2}\right]^{2}=\frac{\left(b_{2} c_{3}-b_{3} c_{2}\right)^{2} \operatorname{det}^{2}[\Lambda]}{c_{3}^{4}} \text {. }
$$

Since $M^{-1}(\mathbf{0})$ is a positive definite matrix, it follows from (24) that $c_{3}>0, \operatorname{det}[U] \neq 0$, and $b_{2} c_{3}-b_{3} c_{2} \neq 0$. From (25), we have $\operatorname{det}[\Lambda]>0$. As a result, linear time-invariant system (22) is controllable. So, the following Riccati matrix equation has a positive definite solution $P$ :

$$
A^{\top} P+P A-P B R^{-1} B^{\top} P=-Q,
$$

where $R>0$ and $Q \in \mathbb{R}^{4 \times 4}$ is a positive definite matrix. From the quadratic optimal control theory, the control law

$$
\tau_{1}=-R^{-1} B^{\top} P,
$$

stabilizes linear system (22) at $\mathbf{x}=\mathbf{0}$ quickly.

From the above statements, we get that the use of the control law $\tau_{1}$ in (18) and (27) in sequence guarantees the global stabilization of reduced-order system (14) at $\mathbf{x}=\mathbf{0}$.

Remark 1. Note that the control law $\tau_{1}$ in (18) is singular when $\mu_{1} \widehat{E}(\mathbf{x})+\mu_{3} g_{1}(\mathbf{x})=0$. This case occurs only when $E(x)<E(0)$ (i.e., $\widehat{E}(\mathbf{x})<0$ ) because $\mu_{1}>0, \mu_{3}>0$, and $g_{1}(\mathbf{x})>0$. When $E(x)<E(0)$, it follows from (1) and (2) that

$$
0>\widehat{E}(\mathbf{x}) \geq\left(\beta_{1}+\beta_{2}\right) \cos x_{2}-E(0) \geq-2 E(0) .
$$

So, we have

$$
\mu_{1} \widehat{E}(\mathbf{x})+\mu_{3} g_{1}(\mathbf{x}) \geq \mu_{3} g_{1}(\mathbf{x})-2 \mu_{1} E(0) .
$$

From (29), it is easy to obtain that the singularity of $\tau_{1}$ in (18) does not occur if

$$
\mu_{3} g_{1}(\mathbf{x})-2 \mu_{1} E(0)>0 .
$$

We select the control parameters such that

$$
\begin{aligned}
& \mu_{3}>\frac{2 \mu_{1} E(0)}{\rho}, \\
& \rho=\min \left\{g_{1}(\mathbf{x})\right\} .
\end{aligned}
$$

This condition guarantees (30) to hold. In other words, the control law in (18) is not singular under condition (31).

Remark 2. There are eight control parameters in this paper, i.e., $r_{1}$ and $r_{2}$ in (12); $\mu_{1}, \mu_{2}$, and $\mu_{3}$ in (16); $\gamma$ in (19); and $R$ and $Q$ in (26). In order to make the control design process clear, a selected guideline for these parameters is given as follows:

(1) An optimal set of $r_{1}$ and $r_{2}$ is selected for (12) that makes the stabilization time of (13b) to be smallest

(2) For fixed $\gamma$, a set of $\mu_{1}, \mu_{2}$, and $\mu_{3}$ is chosen to make reduced-order system (14) enter an arbitrarily small neighbourhood area of $\mathbf{x}=0$

(3) A set of $R$ and $Q$ is selected for the control law $\tau_{1}$ that stabilizes system (22) at the origin

\section{Numerical Example}

A numerical example is presented in this section in order to verify the validity of our presented theoretical results. In the simulation experiment, the physical parameters of the WAcrobot were chosen to be [29]

$$
\left\{\begin{array}{l}
m_{1}=1.22 \mathrm{~kg}, \\
m_{2}=0.28 \mathrm{~kg}, \\
m_{3}=0.72 \mathrm{~kg}, \\
L_{1}=0.05 \mathrm{~m}, \\
L_{2}=0.15 \mathrm{~m}, \\
L_{3}=0.45 \mathrm{~m}, \\
L_{c 2}=0.075 \mathrm{~m}, \\
L_{c 3}=0.225 \mathrm{~m}, \\
J_{1}=0.00153 \mathrm{~kg} \cdot \mathrm{m}^{2}, \\
J_{2}=0.000598 \mathrm{~kg} \cdot \mathrm{m}^{2}, \\
J_{3}=0.013138 \mathrm{~kg} \cdot \mathrm{m}^{2} .
\end{array}\right.
$$

The initial condition was selected to be

$$
\left[\theta_{1}, \theta_{2}, \theta_{3}, \dot{\theta}_{1}, \dot{\theta}_{2}, \dot{\theta}_{3}\right]^{\top}=[0, \pi, 0,0,0,0.1]^{\top} \text {. }
$$

The physical meaning of (33) is that the double inverted pendulum of the WAcrobot begins to move from the straight-down position with a small velocity, while the wheel is stationary at the origin. The control parameters in (12) were selected to be $r_{1}=1$ and $r_{2}=5$. The simulation results of subsystem (13b) are shown in Figure 3 . Note that $\theta_{3}$ and $\theta_{3}$ converge to zero in 0.25 seconds. It means that WAcrobot system (8) changes to reduced-order system (14) quickly. This demonstrates the validity of the presented theoretical results in Section 3.

To show the validity of the stabilizing controller for reduced-order model (14), the parameters in (16) and (18) were chosen as 


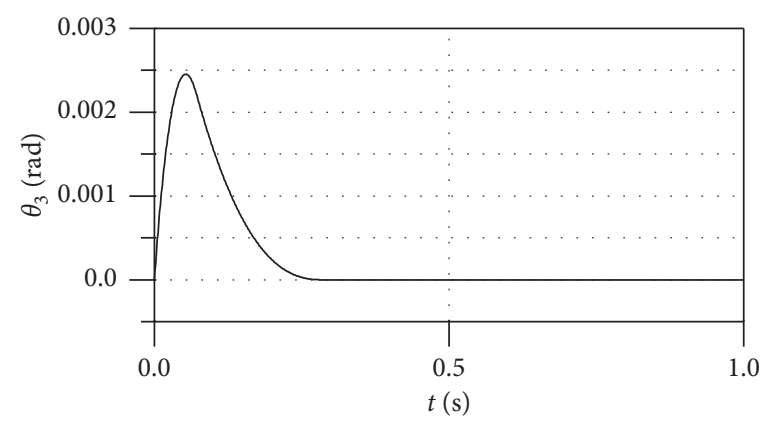

(a)

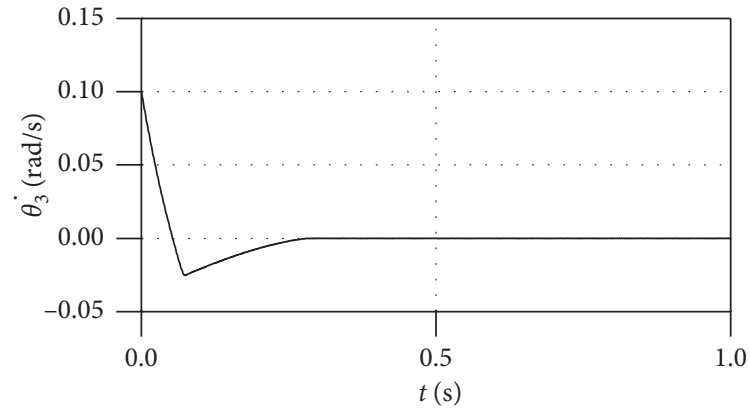

(b)

Figure 3: Time response of $\theta_{3}$ and $\dot{\theta}_{3}$ with $r_{1}=1$ and $r_{2}=5$ in (13b).

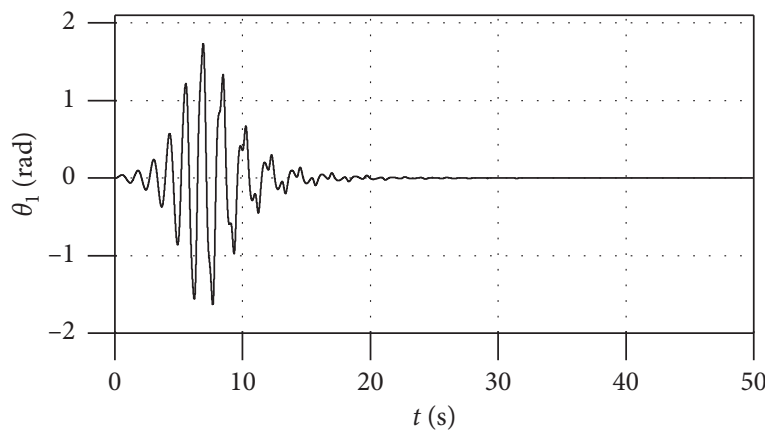

(a)

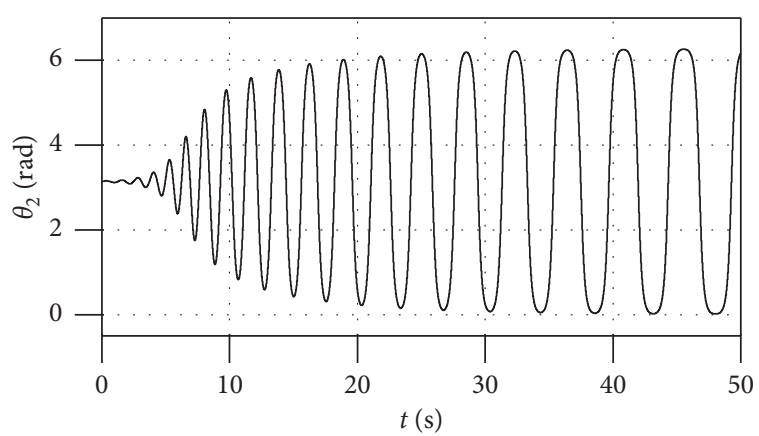

(c)

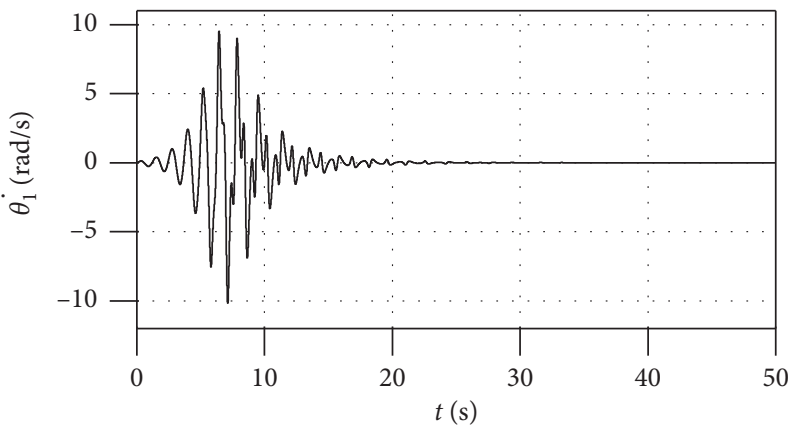

(b)

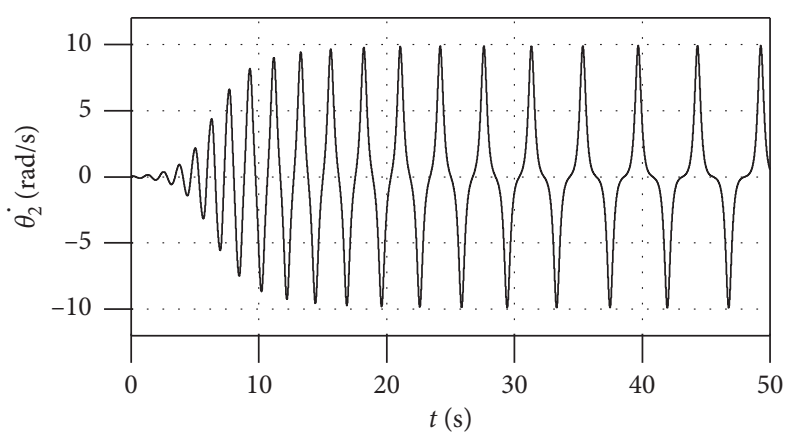

(d)

Figure 4: Time response of $\theta_{1}, \dot{\theta}_{1}, \theta_{2}$, and $\dot{\theta}_{2}$ by controllers (11) and (18).

$$
\left\{\begin{array}{l}
\mu_{1}=350, \\
\mu_{2}=200, \\
\mu_{3}=15, \\
\gamma=50, \\
R=0.5, \\
Q=0.01 I_{4},
\end{array}\right.
$$

where $I_{4}$ is a $4 \times 4$ identity matrix. A simple calculation gives $\rho=141.25$ and $2 \mu_{1} E(0) / \rho=14.147$. Thus, condition (31) is satisfied. Figure 4 shows the simulation results for $\mathbf{x}(t)$ by controllers (11) and (18). It is clear that $\theta_{1}$ and $\dot{\theta}_{1}$ are stabilized at zero while $\theta_{2}$ and $\theta_{2}$ are in a homoclinic orbit. This shows the validity of the theoretical results in Section 4.

When $\mathbf{x}(t)$ enters the small neighbourhood area of $\mathbf{x}(\mathbf{t})=0$ at $t=30 \mathrm{~s}$, the controller $\tau_{1}$ switches to (27) from (18). The simulation results of controllers and $\mathbf{x}(t)$ are shown in Figures 5 and 6, respectively. Note that the switch of $\tau_{1}$ from (18) to (27) causes small sudden changes in $\theta_{1}$ and $\dot{\theta}_{1}$ at $t=30 \mathrm{~s}$. Moreover, it is clear that the WAcrobot can be quickly stabilized at the origin in 40 seconds, and the maximal value of inputs is less than $1.1 \mathrm{Nm}$. Compared with the results in [29], the value of inputs in this paper is more smaller, and the motion process of the WAcrobot system is more smooth and natural. This provides guarantee for the safe and stable operation of the control system. All these 


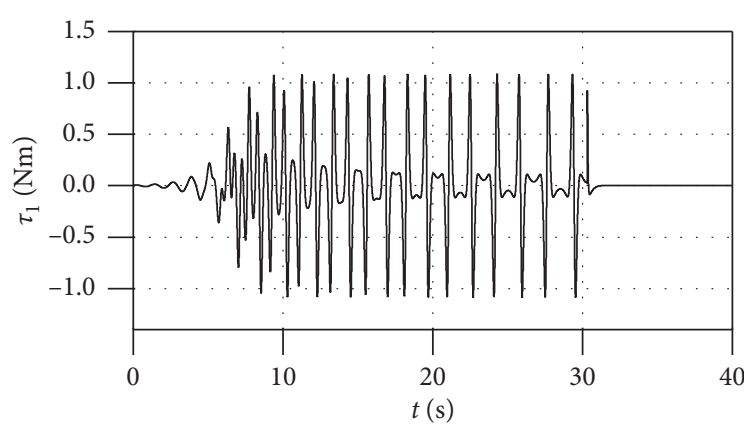

(a)

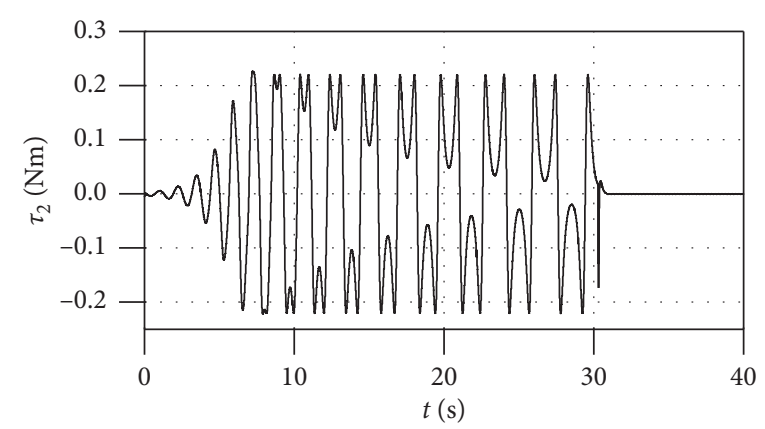

(b)

FIgURE 5: Time response of $\tau_{1}$ and $\tau_{2}$.

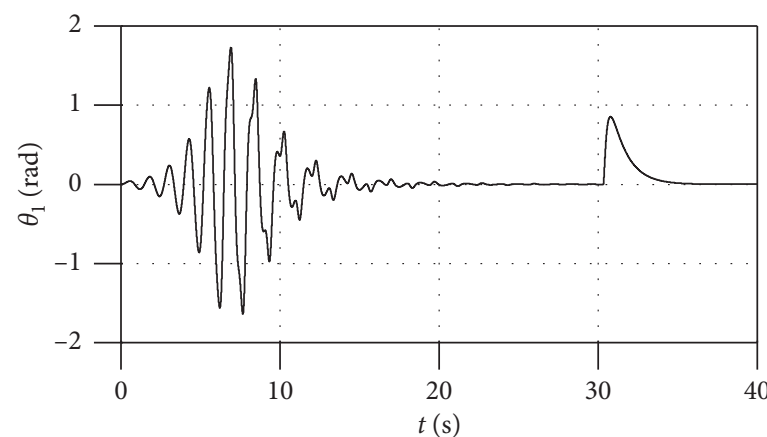

(a)

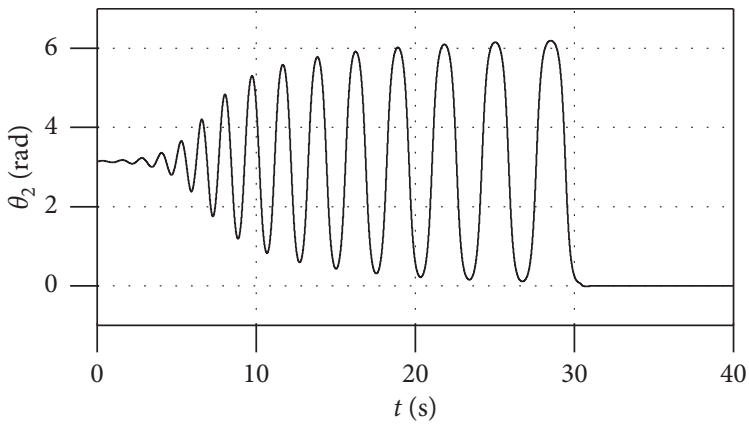

(c)

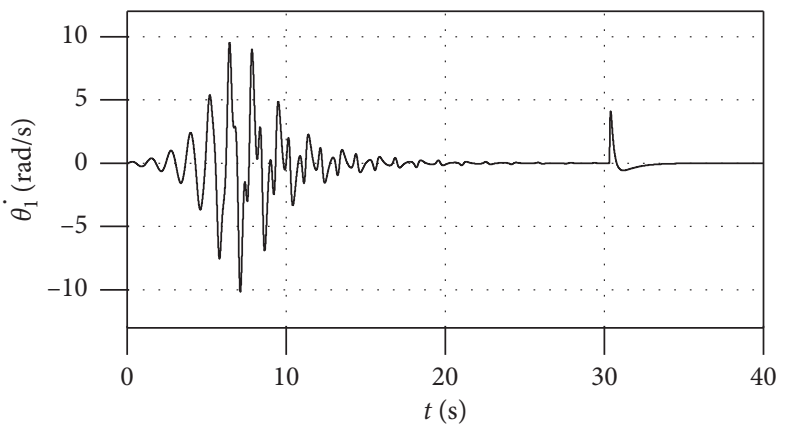

(b)

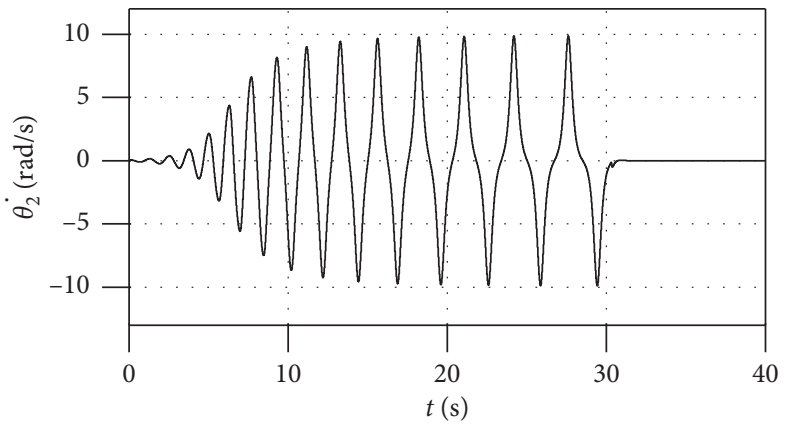

(d)

Figure 6: Time response of $\theta_{1}, \dot{\theta}_{1}, \theta_{2}$, and $\dot{\theta}_{2}$ by controllers (11), (18), and (27).

demonstrate the advantages of our presented control strategy.

\section{Conclusion}

This paper addressed the global stabilization of a 3-DOF underactuated WAcrobot system. A novel stabilizing control strategy was developed. First, the 3-DOF WAcrobot was changed to be a 2-DOF reduced-order model in finite time. This makes the stabilizing control of the WAcrobot be easy to handle. And then, the stabilizing control laws were designed that globally stabilize the reduced-order system at the origin. And the condition that avoids the singularity in the stabilizing control law was also presented. Finally, the numerical simulation example showed the validity of the proposed theoretical results. In the future, we will extend the new strategy to the stabilization of other multi-DOF underactuated systems [31] and will also further explore other stabilizing control methods for the WAcrobot system.

\section{Data Availability}

The data used to support the findings of this study are available from the corresponding author upon request.

\section{Conflicts of Interest}

The authors declare that they have no conflicts of interest.

\section{Acknowledgments}

This work was supported in part by the Natural Science Foundation Program of Shandong Province under Grant no. 
ZR2019YQ28, the Development Plan of Youth Innovation Team of University in Shandong Province under Grant no. 2019KJN007, and the National Natural Science Foundation of China under Grant nos. 61773193, 61703194, 61803194, and 61903171 .

\section{References}

[1] P. Zhao, D.-H. Zhai, Y. Sun, and Y. Li, "Adaptive finite-time control of a class of Markovian jump nonlinear systems with parametric and dynamic uncertainties," Nonlinear Analysis: Hybrid Systems, vol. 29, pp. 234-246, 2018.

[2] C. Jiang, F. Zhang, and T. Li, "Synchronization and antisynchronization of $\mathrm{N}$-coupled fractional-order complex chaotic systems with ring connection," Mathematical Methods in the Applied Sciences, vol. 41, no. 7, pp. 2625-2638, 2018.

[3] X. Yi, R. Guo, and Y. Qi, "Stabilization of chaotic systems with both uncertainty and disturbance by the UDE-based control method," IEEE Access, vol. 8, pp. 62471-62477, 2020.

[4] H. Chen and N. Sun, "Nonlinear control of underactuated systems subject to both actuated and unactuated state constraints with experimental verification," IEEE Transactions on Industrial Electronics, vol. 67, no. 9, pp. 7702-7714, 2019.

[5] N. Sun, D. Liang, Y. Wu, Y. Chen, Y. Qin, and Y. Fang, "Adaptive control for pneumatic artificial muscle systems with parametric uncertainties and unidirectional input constraints," IEEE Transactions on Industrial Informatics, vol. 16, no. 2, pp. 969-979, 2020.

[6] N. Sun, Y. Fu, T. Yang, J. Zhang, Y. Fang, and X. Xin, "Nonlinear motion control of complicated dual rotary crane systems without velocity feedback: design, analysis, and hardware experiments," IEEE Transactions on Automation Science and Engineering, vol. 17, no. 2, pp. 1017-1029, 2020.

[7] Y. Liu and H. Yu, "A survey of underactuated mechanical systems," IET Control Theory \& Applications, vol. 7, no. 7, pp. 921-935, 2013.

[8] W. Chen, C. Xiong, W. Chen, and S. Yue, "Mechanical adaptability analysis of underactuated mechanisms," Robotics and Computer-Integrated Manufacturing, vol. 49, pp. 436447, 2018.

[9] M. Abdeetedal and M. R. Kermani, "Grasp and stress analysis of an underactuated finger for proprioceptive tactile sensing," IEEE/ASME Transactions on Mechatronics, vol. 23, no. 4, pp. 1619-1629, 2018.

[10] G. Oriolo and Y. Nakamura, "Control of mechanical systems with second-order nonholonomic constraints: underactuated manipulators," in Proceedings of the 30th IEEE Conference on Decision and Control, pp. 2398-2403, Brighton, UK, December 1991.

[11] Y. Wang, X. Lai, P. Zhang, and $\mathrm{M}$. Wu, "Adaptive robust control for planar $n$-link underactuated manipulator based on radial basis function neural network and online iterative correction method," Journal of the Franklin Institute, vol. 355, no. 17, pp. 8373-8391, 2018.

[12] X. Xin, "Linear strong structural controllability and observability of an n-link underactuated revolute planar robot with active intermediate joint or joints," Automatica, vol. 94, pp. 436-442, 2018.

[13] N. Sun, Y. Wu, H. Chen, and Y. Fang, "Antiswing cargo transportation of underactuated tower crane systems by a nonlinear controller embedded with an integral term," IEEE Transactions on Automation Science and Engineering, vol. 16, no. 3, pp. 1387-1398, 2019.
[14] A. Zhang, J. She, J. Qiu, C. Yang, and F. Alsaadi, "Design of motion trajectory and tracking control for underactuated cart-pendulum system," International Journal of Robust Nonlinear Control, vol. 29, no. 9, pp. 2458-2470, 2019.

[15] S. Roy and S. Baldi, "Towards structure-independent stabilization for uncertain underactuated Euler-Lagrange systems," Automatica, vol. 113, Article ID 108775, 2020.

[16] X. Lai, Z. Cai, J.-H. She, and Y. Ohyama, "Fuzzy control strategy for acrobots combining model-free and model-based control," IEE Proceedings-Control Theory and Applications, vol. 146, no. 6, pp. 505-510, 1999.

[17] X. Xin, S. Tanaka, J. She, and T. Yamasaki, "New analytical results of energy-based swing-up control for the Pendubot," International Journal of Non-linear Mechanics, vol. 52, pp. 110-118, 2013.

[18] M. Jankovic, D. Fontaine, and P. V. Kokotovic, "TORA example: cascade- and passivity-based control designs," IEEE Transactions on Control Systems Technology, vol. 4, no. 3, pp. 292-297, 1996.

[19] D. Gutirrez, H. Rłos, J. A. Rosales, and R. Galvn-Guerra, "Finite and fixed-time state estimation: pendulum-cart system," IFAC-PapersOnLine, vol. 50, no. 1, pp. 7139-7144, 2017.

[20] M. Spong, "The swing up control problem for the acrobot," IEEE Control Systems Magazine, vol. 15, no. 1, pp. 44-55, 1995.

[21] X. Xin and M. Kaneda, "Analysis of the energy-based swingup control of the Acrobot," International Journal of Robust and Nonlinear Control, vol. 17, no. 16, pp. 1503-1524, 2007.

[22] X. Lai, J. She, S. Yang, and M. Wu, "Comprehensive unified control strategy for underactuated two-link manipulators," IEEE Transactions on Systems Man and Cybernetics Part B, vol. 39, no. 2, pp. 389-398, 2009.

[23] J. She, A. Zhang, X. Lai, and M. Wu, "Global stabilization of 2DOF underactuated mechanical systemsłan equivalent-inputdisturbance approach," Nonlinear Dynamics, vol. 69, no. 1-2, pp. 495-509, 2012.

[24] A. Zhang, X. Lai, M. Wu, and J. She, "Stabilization of underactuated two-link gymnast robot by using trajectory tracking strategy," Applied Mathematics and Computation, vol. 253, pp. 193-204, 2015.

[25] X. Xin and M. Kaneda, "Swing-up control for a 3-DOF gymnastic robot with passive first joint: design and analysis," IEEE Transactions on Robotics, vol. 23, no. 6, pp. 1277-1285, 2007.

[26] A. Zhang, X. Lai, M. Wu, and J. She, "Global stabilization of underactuated spring-coupled three-link horizontal manipulator using position measurements only," Applied Mathematical Modelling, vol. 39, no. 7, pp. 1917-1928, 2015.

[27] Q. Yang and R. Yang, "Nonsingular terminal sliding mode based passive fault-tolerant control of a 3-DOF helicopter system," IFAC-PapersOnLine, vol. 51, no. 24, pp. 1368-1372, 2018.

[28] L. Scalera, A. Gasparetto, and D. Zanotto, "Design and experimental validation of a 3-DOF underactuated pendulumlike robot," IEEE/ASME Transactions on Mechatronics, vol. 25, no. 1, pp. 217-228, 2020.

[29] M. M. Dalvand, B. Shirinzadeh, and S. Nahavandi, "Swing-up and stability control of wheeled Acrobot (WAcrobot)," Automatika, vol. 55, no. 1, pp. 32-40, 2014.

[30] G. He and Z. Geng, "Finite-time stabilization of a comb-drive electrostatic microactuator," IEEE/ASME Transactions on Mechatronics, vol. 17, no. 1, pp. 107-115, 2012.

[31] D. Liu, X. Lai, Y. Wang, X. Wan, and M. Wu, "Position control for planar four-link underactuated manipulator with a passive third joint," ISA Transactions, vol. 87, pp. 46-54, 2019. 